\title{
Mobile Apps for Hematological Conditions: Review and Content Analysis Using the Mobile App Rating Scale
}

Álvaro Narrillos-Moraza ${ }^{1 *}$, PharmD; Patricia Gómez-Martínez-Sagrera ${ }^{*}$, PharmD; Miguel Ángel Amor-García ${ }^{1}$, PharmD; Vicente Escudero-Vilaplana ${ }^{1}$, PharmD, PhD; Roberto Collado-Borrell ${ }^{1}$, PharmD, PhD; Cristina Villanueva-Bueno $^{1}$, PharmD; Ignacio Gómez-Centurión ${ }^{2}, \mathrm{MD}, \mathrm{PhD}$; Ana Herranz-Alonso ${ }^{1}$, PharmD, PhD; María Sanjurjo-Sáez ${ }^{1}$, PharmD

\footnotetext{
${ }^{1}$ Servicio de Farmacia, Hospital General Universitario Gregorio Marañón, Madrid, Spain

${ }^{2}$ Servicio de Hematología, Hospital General Universitario Gregorio Marañón, Madrid, Spain

*these authors contributed equally
}

\section{Corresponding Author:}

Vicente Escudero-Vilaplana, PharmD, PhD

Servicio de Farmacia, Hospital General Universitario Gregorio Marañón

Calle Doctor Esquerdo 43

Madrid, 28007

Spain

Phone: 34915867714

Email: vicente.escudero@salud.madrid.org

\section{Abstract}

Background: Hematological conditions are prevalent disorders that are associated with significant comorbidities and have a major impact on patient care. Concerning new tools for the care of these patients, the number of health apps aimed at hematological patients is growing. Currently, there are no quality analyses or classifications of apps for patients diagnosed with hematological conditions.

Objective: The aim of this study is to analyze the characteristics and quality of apps designed for patients diagnosed with hematological conditions by using the Mobile App Rating Scale (MARS).

Methods: We performed an observational, cross-sectional descriptive study of all smartphone apps for patients diagnosed with hematological conditions. A search was conducted in March 2021 using the following terms: anemia, blood cancer, blood disorder, hematological cancer, hematological malignancy, hematological tumor, hematology, hemophilia, hemorrhage, lymphoma, leukemia, multiple myeloma, thalassemia, thrombocytopenia, and thrombosis. The apps identified were downloaded and evaluated by 2 independent researchers. General characteristics were registered, and quality was analyzed using MARS scores. Interrater reliability was measured by using the Cohen $\kappa$ coefficient.

Results: We identified 2100 apps in the initial search, and 4.19\% (88/2100) of apps met the inclusion criteria and were analyzed. Of the 88 apps, $61 \%$ (54/88) were available on Android, 30\% (26/88) were available on iOS, and 9\% (8/88) were available on both platforms. Moreover, $7 \%$ (6/88) required payment, and 49\% (43/88) were updated in the last year. Only 26\% (23/88) of the apps were developed with the participation of health professionals. Most apps were informative (60/88, 68\%), followed by preventive $(23 / 88,26 \%)$ and diagnostic $(5 / 88,6 \%)$. Most of the apps were intended for patients with anemia (23/88, 26\%). The mean MARS score for the overall quality of the 88 apps was 3.03 (SD 1.14), ranging from 1.19 (lowest-rated app) to 4.86 (highest-rated app). Only 47\% (41/88) of the apps obtained a MARS score of over 3 points (acceptable quality). Functionality was the best-rated section, followed by aesthetics, engagement, information, and app subjective quality. The five apps with the highest MARS score were the following: Multiple Myeloma Manager, Hodgkin Lymphoma Manager, Focus On Lymphoma, ALL Manager, and CLL Manager. The analysis by operating system, developer, and cost revealed statistically significant differences in MARS scores $(P<.001, P<.001$, and $P=.049$, respectively). The interrater agreement between the 2 reviewers was substantial $(\mathrm{k}=0.78)$.

Conclusions: There is great heterogeneity in the quality of apps for patients with hematological conditions. More than half of the apps do not meet acceptable criteria for quality and content. Most of them only provide information about the pathology, lacking interactivity and personalization options. The participation of health professionals in the development of these apps is low, although it is narrowly related to better quality. 
(JMIR Mhealth Uhealth 2022;10(2):e32826) doi: 10.2196/32826

\section{KEYWORDS}

blood; hematology; mHealth; mobile apps; quality; rating tool; mobile phone

\section{Introduction}

\section{Background}

The use of mobile technologies for health is increasing at an unstoppable rate. App capabilities for sharing health care information or real-time patient monitoring make them an important health tool because of their ease of use, broad reach, and wide acceptance [1]. At the beginning of 2021, more than 53,000 medical apps were available in the Android Play Store (one of the main download platforms) [2]. Medical apps have targeted a diverse number of conditions, such as diabetes $[3,4]$, pain [5], rheumatic [6] and psychiatric disorders [7], COVID-19 [8-10], or cancer [11-13]. Apps for patients diagnosed with hematological conditions are also found on the main download platforms, although there is little information about them.

Hematological conditions comprise a wide range of disorders that can be classified as nonmalignant (anemia, hemorrhagic, or thrombotic disorders and conditions affecting blood-forming organs) and malignant (hematological cancers, such as Hodgkin and non-Hodgkin lymphoma, leukemia, or multiple myeloma, among others) [14]. These diseases meet all criteria for qualifying as a very important public health problem, with serious morbidities affecting patients worldwide [14-16]. Many of these conditions, such as hemophilia or anemia, are highly prevalent and become chronic. These patients could benefit from tools that improve treatment adherence or self-management guidelines, making medical apps an increasingly attractive option for this purpose [17,18].

Considering the large number of health apps available for patients with hematological conditions and the increasing interest in tools that encourage patient self-care, a proper review is needed. However, no clear consensus exists as to the appropriate method to assess the quality of health apps [19]. The Mobile App Rating Scale (MARS) is the most widely used scale for evaluating the quality and content of health apps. This allows the evaluation and comparison of apps by relating to their user engagement, functionality, aesthetics, and information quality [20,21]. In addition, it provides a quantitative and validated system that allows both users and health care professionals to avoid unreliable information.

\section{Objective}

The aim of our study is to analyze the characteristics and quality of mobile apps for patients diagnosed with hematological conditions using the MARS.

\section{Methods}

\section{Study Design}

We performed an observational, descriptive, cross-sectional study of all smartphone apps for patients diagnosed with hematological conditions, including hematological malignancies, various types of anemia, and hemorrhagic and thrombotic diathesis, available on the Android and iOS platforms. The study followed the PRISMA-P (Preferred Reporting Items for Systematic Review and Meta-Analysis Protocols) 2015 guidelines for systematic reviews [22].

\section{Eligibility Criteria}

A search on the Apple App Store and Android Play Store was performed in February 2021 by 2 independent health professionals with experience in app analysis, design, and development (PGMS and ANM). The following search terms were used: anemia, blood cancer, blood disorder, hematological cancer, hematological malignancy, hematological tumor, hematology, hemophilia, hemorrhage, lymphoma, leukemia, multiple myeloma, thalassemia, thrombocytopenia, and thrombosis. The reviewers screened the title and download page of the apps. Only apps intended for patients or their caregivers and in English or Spanish were selected. Those apps potentially eligible were downloaded and installed on the appropriate, corresponding mobile device, regardless of the cost. iOS apps were installed on an iPhone 7 (version 14.4.2; Apple Inc) and Android apps on a Nexus 5X (Android version 8.1.0; Google LLC). Apps with nonscientific content; intended for health care professionals; duplicated; not specific for hematological conditions; specific to congresses, meetings, and charitable purposes; and those with restricted access were excluded from the review.

\section{Data Extraction and Quality Assessment}

Apps were individually evaluated in isolation by the same 2 independent reviewers. Variables analyzed were app name, search term (for what the app was found), platform (Android or iOS), developer, hematological disorder, cost, app category (books and reference works, education, entertainment, health and fitness, health and wellness, lifestyle, medicine, simulation, and social media), date of the last update, language, and purpose. Concerning the developer, if hospitals, health authorities, universities, scientific societies, or patients' associations were involved in the design of an app, we classified them as developed by a health organization. The purpose was further classified into the following categories: diagnostic, informative, and preventive depending on whether the priority of the app was to run self-diagnosis, to provide generic data about one or several conditions, or to track treatment and symptoms, respectively. Grading was assessed by the same 2 independent reviewers according to the validated MARS. Data extraction, analysis, and grading were completed within 60 days.

The MARS is a multidimensional instrument that assesses the quality of mobile health apps. The quality assessment consists of a total of 23 items covering 5 dimensions. The dimensions are (1) engagement (5 items: entertainment, interest, customization, interactivity, and target adequacy), (2) functionality (4 items: performance, ease of use, navigation, and gestural design), (3) aesthetics (3 items: layout, graphics, and visual appeal), (4) information quality (7 items: accuracy 
of app description, goals, quality of information, quantity of information, quality of visual information, evidence base, and credibility), and (5) subjective quality (4 items: recommendation, payment willingness, frequency of use, and overall rating). All items were rated on a 5 -point scale (1=inadequate; $2=$ poor; $3=$ acceptable; $4=$ good; $5=$ =xcellent). Then, the overall quality of the app was obtained from the mean score of the domains [20,21].

\section{Data Analysis}

Quantitative variables were expressed as means and SDs and categorical variables as frequencies and percentages. Continuous variables were compared using the 2-tailed $t$ test when the distribution was normal or the Mann-Whitney test when it was not. $\kappa$ coefficient was used to measure the interrater reliability of the data analyzed by the 2 independent researchers [23]. Data were analyzed using Stata (version IC-16; StataCorp). A $P$ value $<.05$ was considered statistically significant.

\section{Results}

\section{Overview}

A total of 2100 apps were retrieved from the Apple App Store and Android Play Store (1661 Android apps and 439 iOS apps). After screening the description and the screenshots available at the app platforms and deleting apps duplicated, 128 apps were selected as potentially eligible. After downloading and checking the fulfillment of the inclusion criteria, 88 apps were finally included in the descriptive analysis. A flow diagram illustrating the selection and exclusion of apps at various stages of the study is shown in Figure 1.

Figure 1. PRISMA (Preferred Reporting Items for Systematic Reviews and Meta-Analyses) flow diagram and app selection. MARS: Mobile App Rating Scale.

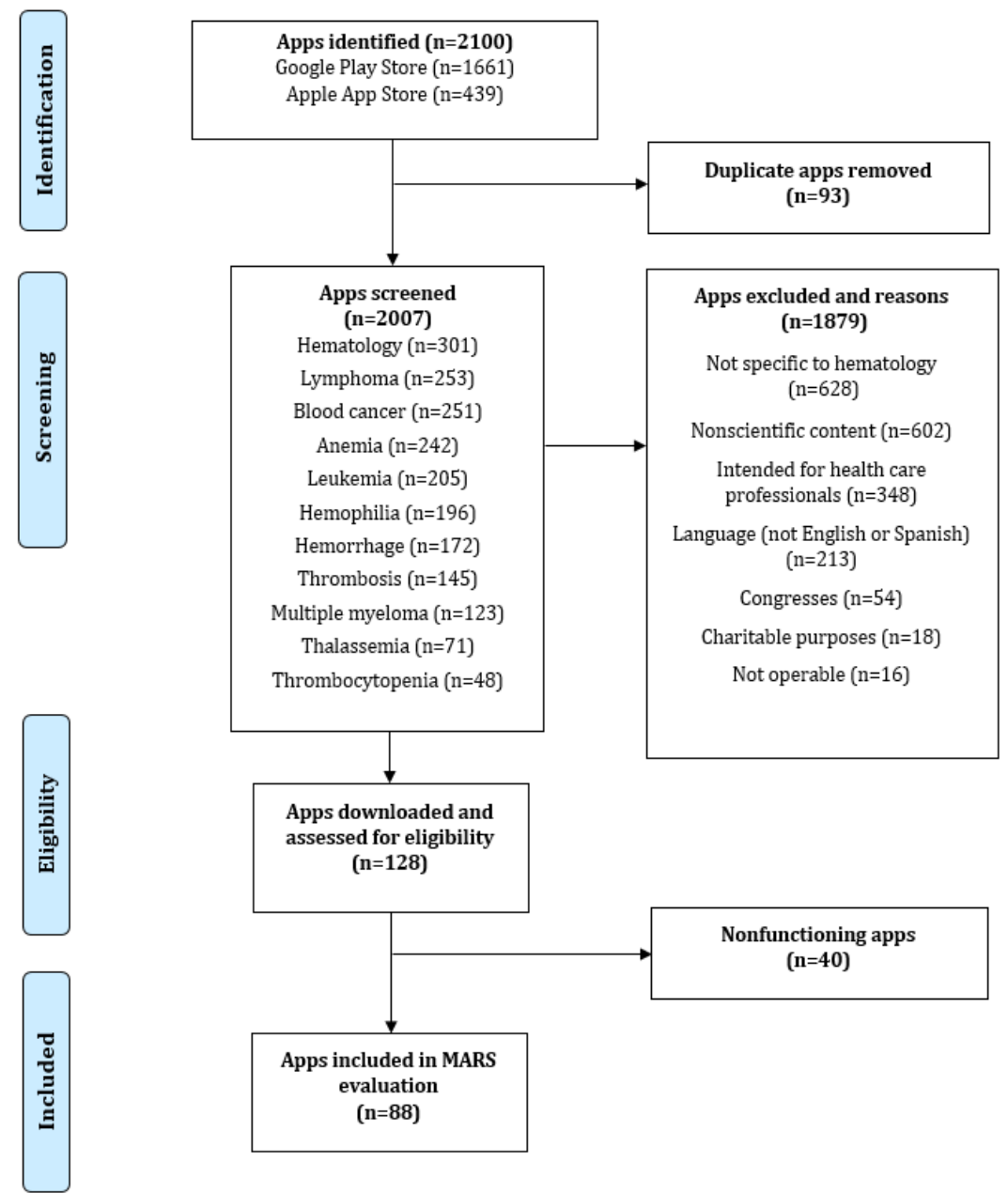

\section{Characteristics and Purposes of Included Apps}

In total, of the 88 apps, 8 (9\%) were found on both digital distribution platforms, whereas $54(61 \%)$ were obtained only from the Android Play Store, and 26 (30\%) were only available at the Apple App Store. In addition, of the 88 apps, only 6 (7\%) required payment (mean cost: mean $€ 3.16$ [US \$3.60], SD $€ 1.57$ [US \$1.79]). Table 1 shows the general characteristics of apps. 
Table 1. General characteristics of the apps.

\begin{tabular}{|c|c|}
\hline Characteristics & Apps, n (\%) \\
\hline \multicolumn{2}{|l|}{ Platform } \\
\hline Android & $54(61)$ \\
\hline iOS & $26(30)$ \\
\hline Android and iOS & $8(9)$ \\
\hline \multicolumn{2}{|l|}{ Cost } \\
\hline No & $82(93)$ \\
\hline Yes & $6(7)$ \\
\hline \multicolumn{2}{|l|}{ Category } \\
\hline Medicine & $35(40)$ \\
\hline Health and wellness & $33(38)$ \\
\hline Health and fitness & $8(9)$ \\
\hline Education & $7(8)$ \\
\hline Books and reference works & $1(1)$ \\
\hline Entertainment & $1(1)$ \\
\hline Lifestyle & $1(1)$ \\
\hline Simulation & $1(1)$ \\
\hline Social media & $1(1)$ \\
\hline \multicolumn{2}{|l|}{ Date of the last update } \\
\hline 2012 & $1(1)$ \\
\hline 2016 & $2(2)$ \\
\hline 2017 & $7(8)$ \\
\hline 2018 & $12(14)$ \\
\hline 2019 & $19(22)$ \\
\hline 2020 & $34(39)$ \\
\hline 2021 & $9(10)$ \\
\hline Not updated & $4(4)$ \\
\hline \multicolumn{2}{|l|}{ Language } \\
\hline English & $80(92)$ \\
\hline Spanish & $4(4)$ \\
\hline English and Spanish & $4(4)$ \\
\hline
\end{tabular}

Regarding purpose, most of the apps were informative (60/88, $68 \%)$, followed by preventive $(23 / 88,26 \%)$ and diagnostic $(5 / 88,6 \%)$. Of the 88 apps, a total of 43 apps $(49 \%)$ were updated in the last year, and 23 apps $(26 \%)$ were designed and developed with the participation of some kind of health care organization. The distribution of apps regarding hematological conditions was anemia $(23 / 88,26 \%)$, leukemia $(12 / 88,14 \%)$, hemophilia $(11 / 88,13 \%)$, thrombosis $(8 / 88,9 \%)$, thalassemia $(7 / 88,8 \%)$, hematological cancers (leukemia, lymphoma, or myeloma; $5 / 88,6 \%)$, hemorrhage $(5 / 88,6 \%)$, lymphoma $(4 / 88$, $5 \%)$, leukemia or lymphoma $(3 / 88,3 \%)$, thrombocytopenia $(3 / 88,3 \%)$, multiple myeloma $(2 / 88,2 \%)$, hematological conditions $(2 / 88,2 \%)$, anemia or hemophilia $(1 / 88,1 \%)$, anemia or thalassemia $(1 / 88,1 \%)$, and hemochromatosis $(1 / 88,1 \%)$. The information on hematological conditions, purpose, app platform, free of cost, updates, developer, and language is shown in Tables 2 and 3. 
Table 2. Characteristics of the apps analyzed. Apps are presented in alphabetical order, from those that start with "A" to those that start with "I."

Name of the app (developer) Hematological disease Purpose Platform $\quad$ Free Updated in the Developed by a last 12 months health organization

\begin{tabular}{|c|c|c|c|c|c|c|c|c|c|c|c|}
\hline & & $\mathrm{I}^{\mathrm{a}}$ & $\mathrm{P}^{\mathrm{b}}$ & $D^{c}$ & iOS & Android & & & & $E^{d}$ & $\mathrm{~S}^{\mathrm{e}}$ \\
\hline $\begin{array}{l}\text { Alimentos para la anemia } \\
\text { (Jotathat) }\end{array}$ & Anemia & $\checkmark$ & & & & $\checkmark$ & $\checkmark$ & $\checkmark$ & & & $\checkmark$ \\
\hline $\begin{array}{l}\text { All Blood Disease and Treat- } \\
\text { ment A-Z (Patrikat Softech) }\end{array}$ & Blood disorders & $\checkmark$ & & & & $\checkmark$ & $\checkmark$ & $\checkmark$ & & $\checkmark$ & \\
\hline ALL Manager (Point of Care) & Leukemia & & $\checkmark$ & & $\checkmark$ & & $\checkmark$ & $\checkmark$ & & $\checkmark$ & \\
\hline ALL Xplained (MedicineX) & Leukemia & $\checkmark$ & & & $\checkmark$ & & $\checkmark$ & & $\checkmark$ & $\checkmark$ & \\
\hline Anemia (Rouseapps) & Anemia & $\checkmark$ & & & & $\checkmark$ & $\checkmark$ & $\checkmark$ & & $\checkmark$ & \\
\hline Anemia (El Makaoui) & Anemia & $\checkmark$ & & & & $\checkmark$ & $\checkmark$ & & & $\checkmark$ & \\
\hline $\begin{array}{l}\text { Anemia Care Diet \& Nutrition } \\
\text { (RecoveryBull) }\end{array}$ & Anemia & $\checkmark$ & & & & $\checkmark$ & $\checkmark$ & $\checkmark$ & & $\checkmark$ & \\
\hline $\begin{array}{l}\text { Anemia Home Remedies } \\
\text { (StatesApps) }\end{array}$ & Anemia & $\checkmark$ & & & & $\checkmark$ & $\checkmark$ & & & $\checkmark$ & \\
\hline $\begin{array}{l}\text { Anemia Home Remedies } \\
\text { (Salim Garba Usman) }\end{array}$ & Anemia & $\checkmark$ & & & & $\checkmark$ & $\checkmark$ & & & $\checkmark$ & \\
\hline Bleeder (Hannes Jung) & Hemophilia & & $\checkmark$ & & $\checkmark$ & & & $\checkmark$ & & $\checkmark$ & \\
\hline $\begin{array}{l}\text { Bleeding After Birth (Jaco } \\
\text { Apps) }\end{array}$ & Hemorrhage & $\checkmark$ & & & & $\checkmark$ & $\checkmark$ & $\checkmark$ & & $\checkmark$ & \\
\hline $\begin{array}{l}\text { Bleeding Disorder } \\
\text { (Koodalappz) }\end{array}$ & Hemorrhage & $\checkmark$ & & & & $\checkmark$ & $\checkmark$ & $\checkmark$ & & $\checkmark$ & \\
\hline $\begin{array}{l}\text { Blood Cancer (Digital Planete } \\
\text { Space) }\end{array}$ & $\begin{array}{l}\text { Hematological can- } \\
\text { cers }\end{array}$ & $\checkmark$ & & & & $\checkmark$ & $\checkmark$ & & & $\checkmark$ & \\
\hline $\begin{array}{l}\text { Blood Cancer Tips (Free } \\
\text { Apps For Everyone) }\end{array}$ & $\begin{array}{l}\text { Hematological can- } \\
\text { cers }\end{array}$ & $\checkmark$ & & & & $\checkmark$ & $\checkmark$ & $\checkmark$ & & $\checkmark$ & \\
\hline $\begin{array}{l}\text { Blood Count Reader free } \\
\text { (Yurii Shevchenko) }\end{array}$ & Anemia & & & $\checkmark$ & & $\checkmark$ & $\checkmark$ & & & $\checkmark$ & \\
\hline $\begin{array}{l}\text { Blood Diseases (Medi- } \\
\text { co_Guide) }\end{array}$ & Blood disorders & $\checkmark$ & & & & $\checkmark$ & $\checkmark$ & & & $\checkmark$ & \\
\hline $\begin{array}{l}\text { Blood Group Genes (Gaurav } \\
\text { Mathur) }\end{array}$ & Anemia or hemophilia & & & $\checkmark$ & $\checkmark$ & & $\checkmark$ & $\checkmark$ & & $\checkmark$ & \\
\hline $\begin{array}{l}\text { Caprini DVT Risk } \\
\text { (NorthShore University } \\
\text { HealthSystem) }\end{array}$ & Thrombosis & $\checkmark$ & & & $\checkmark$ & & $\checkmark$ & & $\checkmark$ & $\checkmark$ & \\
\hline $\begin{array}{l}\text { Childhood Leukemia: A Pre- } \\
\text { ventable Disease (FreeCreativ- } \\
\text { ity2019) }\end{array}$ & Leukemia & $\checkmark$ & & & & $\checkmark$ & $\checkmark$ & $\checkmark$ & & $\checkmark$ & \\
\hline $\begin{array}{l}\text { CIB-Coagulation Interven- } \\
\text { tion Brigade (LFB } \\
\text { Biomedicaments) }\end{array}$ & Hemorrhage & $\checkmark$ & & & $\checkmark$ & & $\checkmark$ & & $\checkmark$ & $\checkmark$ & $\checkmark$ \\
\hline CLL Manager (Point of Care) & Leukemia & & $\checkmark$ & & $\checkmark$ & & $\checkmark$ & $\checkmark$ & & $\checkmark$ & \\
\hline $\begin{array}{l}\text { CLL Watch and Wait Tracker } \\
\text { (Lymphoma Canada) }\end{array}$ & $\begin{array}{l}\text { Leukemia or lym- } \\
\text { phoma }\end{array}$ & & $\checkmark$ & & $\checkmark$ & $\checkmark$ & $\checkmark$ & $\checkmark$ & $\checkmark$ & $\checkmark$ & \\
\hline $\begin{array}{l}\text { CML Life (Incyte Corpora- } \\
\text { tion) }\end{array}$ & Leukemia & $\checkmark$ & & & $\checkmark$ & & $\checkmark$ & & $\checkmark$ & $\checkmark$ & \\
\hline $\begin{array}{l}\text { CML Today (Leukemia Pa- } \\
\text { tient Advocates Foundation) }\end{array}$ & Leukemia & $\checkmark$ & & & $\checkmark$ & & $\checkmark$ & & $\checkmark$ & & $\checkmark$ \\
\hline
\end{tabular}




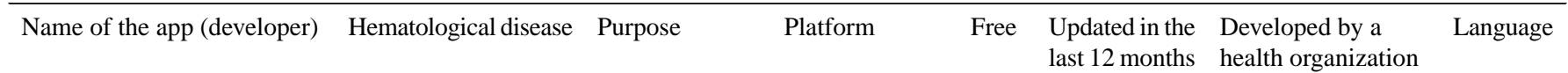

\begin{tabular}{ll}
\hline $\begin{array}{l}\text { Diario de INR (Web Factor } \\
\text { BV) }\end{array}$ & Thrombosis \\
$\begin{array}{l}\text { Don't Walk Alone (Lym- } \\
\text { phoma Canada) }\end{array}$ & Leukemia \\
&
\end{tabular}

Easy Diagnosis-Thalassemia Thalassemia (Sarah Tinmaswala)

EasyCoagLite (Loic Letertre) Thrombosis

Focus On Lymphoma (Lym- Lymphoma phoma Research Foundation)

Folate \& B12 Counter and

Anemia

Tracker (First Line Medical

Communications)

Food For Anemia

Anemia

(MixLabApps)

$\frac{\mathrm{I}^{\mathrm{a}}}{\sqrt{ }} \frac{\mathrm{P}^{\mathrm{b}}}{\mathrm{D}^{\mathrm{c}}}$

last 12 months health organization

HaemActive-Fitness for

People with haemophilia

Hemophilia

(NovoNordisk A/S)

Haemophilia Pal

(Haemophilia Pal)

Hemophilia

Hemo Control (The Simula- Hemophilia tion Crew)

Hemophilia Disease (Bedie- Hemophilia man)

Hemophilia Support (My- Hemophilia HealthTeams)

(1)

Hodgkin Lymphoma Manager Lymphoma (Point of Care)

Home Remedies for Anemia Anemia (Anil Krishna)

$\begin{array}{ll}\begin{array}{l}\text { How To Cure Leukemia } \\ \text { (Apps How To Apps) }\end{array} & \text { Leukemia } \\ \text { iClot (Cranworth Medical } & \text { Thrombosis } \\ \text { Ltd) }\end{array}$

Increase A Low Platelet Thrombocytopenia Count Naturally (Fingerti-

pApps)

INR Care (Nikhil Patel)

Iron Counter and Tracker

Thrombosis

(First Line Medical Communi-

Anemia

cations)

Iron Deficiency Anemia (Be- Anemia dieman)

Iron Tracker-Hemochromato- Hemochromatosis sis (IronTracker)

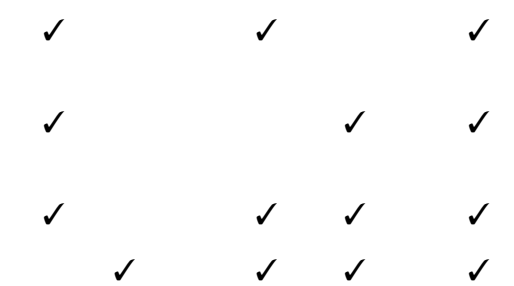

${ }^{a}$ I: informative.

${ }^{b}$ P: preventive.

${ }^{\mathrm{c}} \mathrm{D}$ : diagnostic.

${ }^{\mathrm{d}} \mathrm{E}$ : English.

${ }^{\mathrm{e}} \mathrm{S}$ : Spanish. 
Table 3. Characteristics of the apps analyzed. Apps are presented in alphabetical order, from those that start with "J" to those that start with "Z."

\begin{tabular}{|c|c|c|c|c|c|c|c|c|c|c|c|}
\hline \multirow[t]{2}{*}{ Name of the app (developer) } & \multirow[t]{2}{*}{ Hematological disease } & \multicolumn{3}{|c|}{ Purpose } & \multicolumn{2}{|c|}{ Platform } & \multirow[t]{2}{*}{ Free } & \multirow{2}{*}{$\begin{array}{l}\text { Updated in the } \\
\text { last } 12 \text { months }\end{array}$} & \multirow{2}{*}{$\begin{array}{l}\text { Developed by a } \\
\text { health organization }\end{array}$} & \multicolumn{2}{|c|}{ Language } \\
\hline & & $\mathrm{I}^{\mathrm{a}}$ & $\mathrm{P}^{\mathrm{b}}$ & $D^{c}$ & iOS & Android & & & & $E^{d}$ & $S^{e}$ \\
\hline $\begin{array}{l}\text { Juntos contra la anemia (An- } \\
\text { dres Moran Tello) }\end{array}$ & Anemia & $\checkmark$ & & & & $\checkmark$ & $\checkmark$ & & $\checkmark$ & & $\checkmark$ \\
\hline $\begin{array}{l}\text { Leucemia-Sintomas Y } \\
\text { Tratamiento-FAQ (Things } \\
\text { To Do) }\end{array}$ & Leukemia & $\checkmark$ & & & & $\checkmark$ & $\checkmark$ & & & $\checkmark$ & $\checkmark$ \\
\hline $\begin{array}{l}\text { Leukemia: Symptoms And } \\
\text { Treatment (The Reyv) }\end{array}$ & Leukemia & $\checkmark$ & & & & $\checkmark$ & $\checkmark$ & $\checkmark$ & & $\checkmark$ & \\
\hline $\begin{array}{l}\text { Leukemia Disease (Bedie- } \\
\text { man) }\end{array}$ & Leukemia & $\checkmark$ & & & & $\checkmark$ & $\checkmark$ & & & $\checkmark$ & \\
\hline $\begin{array}{l}\text { Leukemia Disease Treatment } \\
\text { (Woochi Developer) }\end{array}$ & Leukemia & $\checkmark$ & & & & $\checkmark$ & $\checkmark$ & & & $\checkmark$ & \\
\hline $\begin{array}{l}\text { LLS CAR T (The Leukemia } \\
\text { and Lymphoma Society) }\end{array}$ & Blood cancers & $\checkmark$ & & & $\checkmark$ & $\checkmark$ & $\checkmark$ & $\checkmark$ & $\checkmark$ & $\checkmark$ & \\
\hline $\begin{array}{l}\text { LLS Health Manager (The } \\
\text { Leukemia and Lymphoma } \\
\text { Society) }\end{array}$ & Blood cancers & & $\checkmark$ & & $\checkmark$ & $\checkmark$ & $\checkmark$ & $\checkmark$ & $\checkmark$ & $\checkmark$ & \\
\hline $\begin{array}{l}\text { LRF Understanding Lym- } \\
\text { phoma (Lymphoma Research } \\
\text { Foundation) }\end{array}$ & Lymphoma & $\checkmark$ & & & & $\checkmark$ & $\checkmark$ & $\checkmark$ & $\checkmark$ & $\checkmark$ & \\
\hline $\begin{array}{l}\text { LRFFactSheets (Lymphoma } \\
\text { Research Foundation) }\end{array}$ & Lymphoma & $\checkmark$ & & & $\checkmark$ & & $\checkmark$ & $\checkmark$ & $\checkmark$ & $\checkmark$ & \\
\hline $\begin{array}{l}\text { Microhealth Hemofilia (Micro- } \\
\text { Health LLC) }\end{array}$ & Hemophilia & & $\checkmark$ & & $\checkmark$ & $\checkmark$ & $\checkmark$ & $\checkmark$ & $\checkmark$ & $\checkmark$ & $\checkmark$ \\
\hline $\begin{array}{l}\text { Mi Hemofilia (Rogelio Rob- } \\
\text { les Tarano) }\end{array}$ & Hemophilia & $\checkmark$ & & & & $\checkmark$ & $\checkmark$ & & $\checkmark$ & $\checkmark$ & \\
\hline $\begin{array}{l}\text { Multiple Myeloma Manager } \\
\text { (Point of Care) }\end{array}$ & Multiple myeloma & & $\checkmark$ & & $\checkmark$ & & $\checkmark$ & $\checkmark$ & & $\checkmark$ & \\
\hline $\begin{array}{l}\text { My Blood Count (Sean Bot- } \\
\text { tomley) }\end{array}$ & Anemia & & $\checkmark$ & & $\checkmark$ & & $\checkmark$ & $\checkmark$ & & $\checkmark$ & \\
\hline My HHT Tracker (Cure HHT) & Hemochromatosis & & $\checkmark$ & & $\checkmark$ & & $\checkmark$ & & & $\checkmark$ & \\
\hline My INR (iMonitorMy) & Thrombosis & & $\checkmark$ & & & $\checkmark$ & $\checkmark$ & & & $\checkmark$ & \\
\hline $\begin{array}{l}\text { My Iron Manager (Good Dog } \\
\text { Design Pty Ltd) }\end{array}$ & Anemia & & $\checkmark$ & & & $\checkmark$ & $\checkmark$ & $\checkmark$ & & $\checkmark$ & \\
\hline $\begin{array}{l}\text { Myeloma Cancer Guide (Ev- } \\
\text { eryone Learning Apps) }\end{array}$ & Multiple myeloma & $\checkmark$ & & & & $\checkmark$ & $\checkmark$ & $\checkmark$ & & $\checkmark$ & \\
\hline $\begin{array}{l}\text { myPROBE (Design2Code } \\
\text { Inc) }\end{array}$ & Hemophilia & & $\checkmark$ & & $\checkmark$ & & $\checkmark$ & $\checkmark$ & & $\checkmark$ & \\
\hline $\begin{array}{l}\text { myWAPPS (Design2Code } \\
\text { Inc) }\end{array}$ & Hemophilia & & $\checkmark$ & & $\checkmark$ & & $\checkmark$ & $\checkmark$ & & $\checkmark$ & \\
\hline $\begin{array}{l}\text { NCCN Patient Guides for } \\
\text { Cancer (National Comprehen- } \\
\text { sive Cancer Network) }\end{array}$ & Blood cancers & $\checkmark$ & & & & $\checkmark$ & $\checkmark$ & $\checkmark$ & $\checkmark$ & $\checkmark$ & \\
\hline $\begin{array}{l}\text { PA Pernicious Anaemia (B12 } \\
\text { Global Limited) }\end{array}$ & Anemia & & $\checkmark$ & & $\checkmark$ & & & & & $\checkmark$ & \\
\hline $\begin{array}{l}\text { Pernicious-Anemia Advice } \\
\text { (MoreFlow) }\end{array}$ & Anemia & $\checkmark$ & & & & $\checkmark$ & $\checkmark$ & $\checkmark$ & & $\checkmark$ & \\
\hline $\begin{array}{l}\text { Pregnancy \& Anaemia (Fu- } \\
\text { mo) }\end{array}$ & Anemia & $\checkmark$ & & & & $\checkmark$ & $\checkmark$ & $\checkmark$ & & $\checkmark$ & \\
\hline
\end{tabular}




\begin{tabular}{|c|c|c|c|c|c|c|c|c|c|c|c|}
\hline \multirow[t]{2}{*}{ Name of the app (developer) } & \multirow[t]{2}{*}{ Hematological disease } & \multicolumn{3}{|c|}{ Purpose } & \multicolumn{2}{|c|}{ Platform } & \multirow[t]{2}{*}{ Free } & \multirow{2}{*}{$\begin{array}{l}\text { Updated in the } \\
\text { last } 12 \text { months }\end{array}$} & \multirow{2}{*}{$\begin{array}{l}\text { Developed by a } \\
\text { health organization }\end{array}$} & \multicolumn{2}{|c|}{ Language } \\
\hline & & $\mathrm{I}^{\mathrm{a}}$ & $\mathrm{P}^{\mathrm{b}}$ & $D^{c}$ & iOS & Android & & & & $E^{d}$ & $S^{e}$ \\
\hline $\begin{array}{l}\text { Recetas y consejos para com- } \\
\text { batir la anemia (App Free En- } \\
\text { joy) }\end{array}$ & Anemia & & $\checkmark$ & & & $\checkmark$ & $\checkmark$ & & & & $\checkmark$ \\
\hline $\begin{array}{l}\text { Recognize Anemia Disease } \\
\text { (Media Clinic) }\end{array}$ & Anemia & $\checkmark$ & & & & $\checkmark$ & $\checkmark$ & & & $\checkmark$ & \\
\hline $\begin{array}{l}\text { Recognize Hemophilia Dis- } \\
\text { ease (Media Clinic) }\end{array}$ & Hemophilia & $\checkmark$ & & & & $\checkmark$ & $\checkmark$ & & & $\checkmark$ & \\
\hline $\begin{array}{l}\text { Recognize Thalassemia Dis- } \\
\text { ease (Media Clinic) }\end{array}$ & Thalassemia & $\checkmark$ & & & & $\checkmark$ & $\checkmark$ & & & $\checkmark$ & \\
\hline $\begin{array}{l}\text { Recognize Thrombocytopenia } \\
\text { (Media Clinic) }\end{array}$ & Thrombocytopenia & $\checkmark$ & & & & $\checkmark$ & $\checkmark$ & & & $\checkmark$ & \\
\hline Sickle Cell Anemia (Fumo) & Anemia & $\checkmark$ & & & & $\checkmark$ & $\checkmark$ & $\checkmark$ & & $\checkmark$ & \\
\hline $\begin{array}{l}\text { Sickle Cell Anemia Home } \\
\text { remedy (JGWS) }\end{array}$ & Anemia & & $\checkmark$ & & & $\checkmark$ & $\checkmark$ & & & $\checkmark$ & \\
\hline $\begin{array}{l}\text { Sickle Cell Disease (Kabi- } \\
\text { rapp) }\end{array}$ & Anemia & $\checkmark$ & & & & $\checkmark$ & $\checkmark$ & & & $\checkmark$ & \\
\hline $\begin{array}{l}\text { STB-Stop The Bleed (Uni- } \\
\text { formed Services University) }\end{array}$ & Hemorrhage & $\checkmark$ & & & $\checkmark$ & & $\checkmark$ & & $\checkmark$ & $\checkmark$ & \\
\hline SUSOKA (Subrata Saha) & Thalassemia & & & $\checkmark$ & & $\checkmark$ & $\checkmark$ & $\checkmark$ & & $\checkmark$ & \\
\hline $\begin{array}{l}\text { Thalassemia Early Detection } \\
\text { (ILIANA) }\end{array}$ & Thalassemia & & & $\checkmark$ & & $\checkmark$ & $\checkmark$ & $\checkmark$ & & $\checkmark$ & \\
\hline $\begin{array}{l}\text { Thalassemia Disease (Bedie- } \\
\text { man) }\end{array}$ & Thalassemia & $\checkmark$ & & & & $\checkmark$ & $\checkmark$ & & & $\checkmark$ & \\
\hline $\begin{array}{l}\text { ThaliMe (Curatio Networks } \\
\text { Inc) }\end{array}$ & Thalassemia & $\checkmark$ & & & & $\checkmark$ & $\checkmark$ & & & $\checkmark$ & \\
\hline $\begin{array}{l}\text { thalTracker (University } \\
\text { Health Network) }\end{array}$ & Thalassemia & $\checkmark$ & & & $\checkmark$ & & $\checkmark$ & & & $\checkmark$ & \\
\hline $\begin{array}{l}\text { The Cancer App (Interactive } \\
\text { Pharma solutions limited) }\end{array}$ & Blood cancers & $\checkmark$ & & & $\checkmark$ & & $\checkmark$ & $\checkmark$ & $\checkmark$ & $\checkmark$ & \\
\hline $\begin{array}{l}\text { The Seven Types of Anemia } \\
\text { (Mrbeli) }\end{array}$ & $\begin{array}{l}\text { Thalassemia or ane- } \\
\text { mia }\end{array}$ & $\checkmark$ & & & & $\checkmark$ & $\checkmark$ & & & $\checkmark$ & \\
\hline $\begin{array}{l}\text { Transplant Guidelines (Nation- } \\
\text { al Marrow Donor Program/Be } \\
\text { The Match) }\end{array}$ & Blood cancers & $\checkmark$ & & & & $\checkmark$ & $\checkmark$ & & $\checkmark$ & $\checkmark$ & \\
\hline $\begin{array}{l}\text { Trombocytopenia Disease } \\
\text { (Bedieman) }\end{array}$ & Thrombocytopenia & $\checkmark$ & & & & $\checkmark$ & $\checkmark$ & & & $\checkmark$ & \\
\hline $\begin{array}{l}\text { VTE Calc (Lindum Medical } \\
\text { Ltd) }\end{array}$ & Thrombosis & $\checkmark$ & & & $\checkmark$ & & $\checkmark$ & & & $\checkmark$ & \\
\hline
\end{tabular}

${ }^{\mathrm{a}} \mathrm{I}$ : informative.

${ }^{\mathrm{b}} \mathrm{P}$ : preventive.

${ }^{\mathrm{c}} \mathrm{D}$ : diagnostic.

${ }^{\mathrm{d}}$ E: English.

${ }^{\mathrm{e}} \mathrm{S}$ : Spanish.

\section{Rating of Apps on the MARS}

The specific MARS ratings for each app are shown in Tables 4 and 5. The mean score for the overall quality was 3.03 (SD 1.14), ranging from 1.19 (lowest rated app) to 4.86 (highest rated app). On average, the best-rated section was functionality (mean 3.44, SD 1.07), followed by aesthetics (mean 3.10, SD 1.23 ), engagement (mean 3.06, SD 1.32), information (mean 2.95, SD 1.09), and app subjective quality (mean 2.61, SD 1.28). 
Table 4. Mobile App Rating Scale scores of the evaluated apps (rating out of 5). The first half $(41 / 88,47 \%)$ of the apps are presented here.

\begin{tabular}{|c|c|c|c|c|c|c|}
\hline Name of the app (developer) & $\begin{array}{l}\text { Engagement, } \\
\text { score }\end{array}$ & $\begin{array}{l}\text { Functionality, } \\
\text { score }\end{array}$ & $\begin{array}{l}\text { Aesthetics, } \\
\text { score }\end{array}$ & $\begin{array}{l}\text { Information, } \\
\text { score }\end{array}$ & $\begin{array}{l}\text { Subjective quality, } \\
\text { score }\end{array}$ & Overall \\
\hline Multiple Myeloma Manager (Point of Care) & 5.00 & 5.00 & 5.00 & 4.57 & 4.75 & 4.86 \\
\hline Hodgkin Lymphoma Manager (Point of Care) & 5.00 & 5.00 & 5.00 & 4.43 & 4.75 & 4.84 \\
\hline $\begin{array}{l}\text { Focus On Lymphoma (Lymphoma Research } \\
\text { Foundation) }\end{array}$ & 4.90 & 4.88 & 4.83 & 4.43 & 5.00 & 4.81 \\
\hline ALL Manager (Point of Care) & 5.00 & 4.88 & 4.67 & 4.43 & 4.88 & 4.77 \\
\hline CLL Manager (Point of Care) & 5.00 & 4.75 & 4.67 & 4.43 & 4.75 & 4.72 \\
\hline $\begin{array}{l}\text { Transplant Guidelines (National Marrow Donor } \\
\text { Program/Be The Match) }\end{array}$ & 4.80 & 5.00 & 4.50 & 4.43 & 4.50 & 4.65 \\
\hline $\begin{array}{l}\text { HaemActive-Fitness for people with } \\
\text { haemophilia (NovoNordisk A/S) }\end{array}$ & 4.90 & 4.38 & 5.00 & 4.57 & 4.38 & 4.64 \\
\hline Mi Hemofilia (Rogelio Robles Tarano) & 4.30 & 4.63 & 4.83 & 4.79 & 4.63 & 4.63 \\
\hline My INR (iMonitorMy) & 4.60 & 4.88 & 4.17 & 4.64 & 4.38 & 4.53 \\
\hline My Iron Manager (Good Dog Design Pty Ltd) & 4.60 & 4.50 & 4.50 & 4.71 & 4.13 & 4.49 \\
\hline myWAPPS (Design2Code Inc) & 4.90 & 4.88 & 4.67 & 4.07 & 3.88 & 4.48 \\
\hline $\begin{array}{l}\text { CLL Watch and Wait Tracker (Lymphoma } \\
\text { Canada) }\end{array}$ & 4.60 & 4.00 & 4.67 & 4.36 & 4.75 & 4.47 \\
\hline Bleeder (Hannes Jung) & 4.70 & 4.50 & 4.50 & 4.14 & 4.50 & 4.47 \\
\hline Microhealth Hemofilia (MicroHealth LLC) & 5.00 & 4.50 & 4.17 & 4.21 & 4.25 & 4.43 \\
\hline Iron Tracker-Hemochromatosis (IronTracker) & 4.40 & 4.63 & 4.67 & 4.43 & 4.00 & 4.42 \\
\hline $\begin{array}{l}\text { STB-Stop The Bleed (Uniformed Services Uni- } \\
\text { versity) }\end{array}$ & 4.00 & 4.63 & 4.33 & 4.64 & 4.50 & 4.42 \\
\hline PA Pernicious Anaemia (B12 Global Limited) & 4.70 & 4.50 & 4.50 & 4.14 & 4.13 & 4.39 \\
\hline Hemophilia Pal (Haemophilia Pal) & 4.50 & 4.38 & 4.17 & 4.43 & 4.38 & 4.37 \\
\hline ThaliMe (Curatio Networks Inc) & 4.60 & 4.50 & 4.67 & 3.79 & 3.88 & 4.29 \\
\hline My HHT Tracker (Cure HHT) & 4.70 & 4.63 & 4.50 & 3.43 & 4.13 & 4.28 \\
\hline CML Life (Incyte Corporation) & 4.40 & 4.75 & 4.67 & 3.71 & 3.63 & 4.23 \\
\hline INR Care (Nikhil Patel) & 4.50 & 4.38 & 4.67 & 3.29 & 4.13 & 4.19 \\
\hline Diario de INR (Web Factor BV) & 4.60 & 4.13 & 4.67 & 3.93 & 3.63 & 4.19 \\
\hline $\begin{array}{l}\text { NCCN Patient Guides for Cancer (National } \\
\text { Comprehensive Cancer Network) }\end{array}$ & 3.10 & 4.75 & 4.67 & 4.36 & 4.00 & 4.17 \\
\hline My Blood Count (Sean Bottomley) & 4.80 & 4.38 & 4.50 & 3.64 & 3.50 & 4.16 \\
\hline $\begin{array}{l}\text { LLS Health Manager (The Leukemia and Lym- } \\
\text { phoma Society) }\end{array}$ & 4.70 & 4.00 & 3.83 & 4.21 & 4.00 & 4.15 \\
\hline thalTracker (University Health Network) & 4.60 & 4.50 & 4.50 & 3.86 & 3.25 & 4.14 \\
\hline Don't Walk Alone (Lymphoma Canada) & 4.90 & 3.38 & 4.17 & 4.21 & 3.38 & 4.01 \\
\hline $\begin{array}{l}\text { The Cancer App (Interactive Pharma Solutions } \\
\text { Limited) }\end{array}$ & 4.30 & 4.25 & 4.50 & 3.71 & 3.13 & 3.98 \\
\hline Hemophilia Support (MyHealthTeams) & 4.60 & 3.75 & 3.67 & 3.14 & 4.50 & 3.93 \\
\hline $\begin{array}{l}\text { CML Today (Leukemia Patient Advocates Foun- } \\
\text { dation) }\end{array}$ & 3.70 & 4.38 & 3.50 & 4.29 & 3.63 & 3.90 \\
\hline Pernicious Anemia Advice (MoreFlow) & 3.10 & 4.50 & 4.33 & 4.00 & 3.50 & 3.89 \\
\hline ALL Xplained (MedicineX) & 3.10 & 4.38 & 4.00 & 3.71 & 2.75 & 3.59 \\
\hline VTE Calc (Lindum Medical Ltd) & 3.80 & 4.38 & 3.17 & 3.86 & 2.63 & 3.56 \\
\hline myPROBE (Design2Code Inc) & 3.80 & 4.25 & 4.00 & 2.71 & 2.13 & 3.38 \\
\hline
\end{tabular}




\begin{tabular}{|c|c|c|c|c|c|c|}
\hline Name of the app (developer) & $\begin{array}{l}\text { Engagement, } \\
\text { score }\end{array}$ & $\begin{array}{l}\text { Functionality, } \\
\text { score }\end{array}$ & $\begin{array}{l}\text { Aesthetics, } \\
\text { score }\end{array}$ & $\begin{array}{l}\text { Information, } \\
\text { score }\end{array}$ & $\begin{array}{l}\text { Subjective quality, } \\
\text { score }\end{array}$ & Overall \\
\hline Alimentos para la anemia (Jotathat) & 2.70 & 3.13 & 3.50 & 3.43 & 3.13 & 3.18 \\
\hline $\begin{array}{l}\text { Folate \& B12 Counter and Tracker (First Line } \\
\text { Medical Communications) }\end{array}$ & 3.10 & 4.13 & 2.50 & 3.36 & 2.63 & 3.14 \\
\hline Blood Group Genes (Gaurav Mathur) & 4.20 & 3.25 & 4.00 & 2.21 & 2.00 & 3.13 \\
\hline $\begin{array}{l}\text { Iron Counter and Tracker (First Line Medical } \\
\text { Communications) }\end{array}$ & 3.10 & 4.13 & 2.50 & 3.36 & 2.50 & 3.12 \\
\hline $\begin{array}{l}\text { All Blood Disease and Treatment A-Z (Patrikat } \\
\text { Softech) }\end{array}$ & 2.60 & 3.88 & 3.00 & 3.21 & 2.50 & 3.04 \\
\hline $\begin{array}{l}\text { LRF Understanding Lymphoma (Lymphoma Re- } \\
\text { search Foundation) }\end{array}$ & 3.10 & 3.38 & 3.33 & 3.36 & 2.00 & 3.03 \\
\hline
\end{tabular}


Table 5. Mobile App Rating Scale scores of the evaluated apps (rating out of 5). The second half (47/88, 53\%) of the apps are presented here.

\begin{tabular}{|c|c|c|c|c|c|c|}
\hline Name of the app (developer) & $\begin{array}{l}\text { Engagement, } \\
\text { score }\end{array}$ & $\begin{array}{l}\text { Functionality, } \\
\text { score }\end{array}$ & $\begin{array}{l}\text { Aesthetics, } \\
\text { score }\end{array}$ & $\begin{array}{l}\text { Information, } \\
\text { score }\end{array}$ & $\begin{array}{l}\text { Subjective quality, } \\
\text { score }\end{array}$ & Overall \\
\hline LRFFactSheets (Lymphoma Research Foundation) & 3.40 & 3.38 & 3.17 & 3.14 & 1.88 & 2.99 \\
\hline Juntos contra la anemia (Andres Moran Tello) & 3.00 & 3.13 & 3.17 & 3.07 & 2.38 & 2.95 \\
\hline EasyCoagLite (Loic Letertre) & 3.30 & 2.88 & 3.00 & 2.79 & 2.50 & 2.89 \\
\hline Hemo Control (The Simulation Crew) & 3.60 & 3.00 & 3.50 & 2.07 & 1.88 & 2.81 \\
\hline $\begin{array}{l}\text { Caprini DVT Risk (NorthShore University } \\
\text { HealthSystem) }\end{array}$ & 3.70 & 3.38 & 2.67 & 2.57 & 1.38 & 2.74 \\
\hline Recognize Thrombocytopenia (Media Clinic) & 2.30 & 3.88 & 2.00 & 2.50 & 1.75 & 2.49 \\
\hline Thalasemia Early Detection (Iliana) & 3.00 & 3.25 & 2.67 & 2.21 & 1.25 & 2.48 \\
\hline Recognize Hemophilia Disease (Media Clinic) & 2.30 & 3.88 & 1.67 & 2.50 & 1.88 & 2.44 \\
\hline Sickle Cell Anemia Home remedy (JGWS) & 1.80 & 2.63 & 3.00 & 2.50 & 2.25 & 2.44 \\
\hline Recognize Thalassemia Disease (Media Clinic) & 2.30 & 3.88 & 1.67 & 2.50 & 1.75 & 2.42 \\
\hline $\begin{array}{l}\text { Increase A Low Platelet Count Naturally (Finger- } \\
\text { tipApps) }\end{array}$ & 1.80 & 3.38 & 2.33 & 2.43 & 2.13 & 2.41 \\
\hline Recognize Anemia Disease (Media Clinic) & 2.30 & 3.25 & 2.17 & 2.50 & 1.75 & 2.39 \\
\hline Anemia (RouseApps) & 2.00 & 3.38 & 2.00 & 2.57 & 1.88 & 2.36 \\
\hline $\begin{array}{l}\text { LLS CAR T (The Leukemia and Lymphoma So- } \\
\text { ciety) }\end{array}$ & 2.40 & 3.25 & 2.83 & 2.07 & 1.25 & 2.36 \\
\hline $\begin{array}{l}\text { Blood Clot Home \& Natural Remedies (Salim } \\
\text { Garba Usman) }\end{array}$ & 2.00 & 3.25 & 2.67 & 2.21 & 1.63 & 2.35 \\
\hline Anemia Home Remedies (StatesApps) & 1.80 & 3.63 & 2.17 & 2.29 & 1.75 & 2.33 \\
\hline Bleeding After Birth (JacoApps) & 1.90 & 3.13 & 2.83 & 2.14 & 1.63 & 2.33 \\
\hline The Seven Types of Anemia (MrBeli) & 2.20 & 3.25 & 2.00 & 2.36 & 1.75 & 2.31 \\
\hline Sickle Cell Disease (Kabirapp) & 1.90 & 2.38 & 2.83 & 2.00 & 2.25 & 2.27 \\
\hline Bleeding Disorder (Koodalappz) & 2.10 & 2.50 & 2.83 & 2.29 & 1.63 & 2.27 \\
\hline Blood Diseases (Medico_Guide) & 1.80 & 2.63 & 2.27 & 2.29 & 2.00 & 2.18 \\
\hline $\begin{array}{l}\text { Childhood Leukemia: A Preventable Disease } \\
\text { (FreeCreativity2019) }\end{array}$ & 1.80 & 2.63 & 1.83 & 2.21 & 2.38 & 2.17 \\
\hline Trombocytopenia Disease (Bedieman) & 1.80 & 2.75 & 2.00 & 2.50 & 1.75 & 2.16 \\
\hline Food For Anemia (MixLabApps) & 1.80 & 3.13 & 2.33 & 2.14 & 1.38 & 2.16 \\
\hline Iron Deficiency Anemia (Bedieman) & 1.80 & 3.00 & 2.00 & 2.21 & 1.75 & 2.15 \\
\hline Hemophilia Disease (Bedieman) & 1.80 & 2.75 & 2.00 & 2.50 & 1.63 & 2.14 \\
\hline Thalassemia Disease (Bedieman) & 1.70 & 2.75 & 2.00 & 2.50 & 1.63 & 2.12 \\
\hline $\begin{array}{l}\text { Easy Diagnosis_-Thalassemia (Sarah Tin- } \\
\text { maswala) }\end{array}$ & 1.90 & 3.25 & 1.67 & 2.00 & 1.38 & 2.04 \\
\hline Home Remedies for Anemia (Anil Krishna) & 1.80 & 2.63 & 2.17 & 1.93 & 1.63 & 2.03 \\
\hline SUSOKA (Subrata Saha) & 2.40 & 2.38 & 2.17 & 1.57 & 1.38 & 1.98 \\
\hline $\begin{array}{l}\text { Leucemia_Sintomas Y Tratamiento-FAQ } \\
\text { (Things To Do) }\end{array}$ & 1.60 & 2.13 & 2.00 & 2.43 & 1.50 & 1.93 \\
\hline Anemia Care Diet \& Nutrition (RecoveryBull) & 2.00 & 2.13 & 2.00 & 2.00 & 1.50 & 1.93 \\
\hline Sickle Cell Anemia (Fumo) & 1.80 & 2.38 & 1.83 & 1.79 & 1.63 & 1.88 \\
\hline iClot (Cranworth Medical Ltd) & 1.90 & 2.00 & 2.17 & 1.93 & 1.38 & 1.87 \\
\hline Pregnancy \& Anaemia (Fumo) & 1.80 & 2.38 & 2.00 & 1.79 & 1.38 & 1.87 \\
\hline Anemia (El Makaoui) & 1.50 & 2.75 & 2.00 & 1.64 & 1.25 & 1.83 \\
\hline Anemia Home Remedies (Salim Garba Usman) & 1.80 & 2.00 & 1.83 & 1.86 & 1.63 & 1.82 \\
\hline
\end{tabular}




\begin{tabular}{|c|c|c|c|c|c|c|}
\hline Name of the app (developer) & $\begin{array}{l}\text { Engagement, } \\
\text { score }\end{array}$ & $\begin{array}{l}\text { Functionality, } \\
\text { score }\end{array}$ & $\begin{array}{l}\text { Aesthetics, } \\
\text { score }\end{array}$ & $\begin{array}{l}\text { Information, } \\
\text { score }\end{array}$ & $\begin{array}{l}\text { Subjective quality, } \\
\text { score }\end{array}$ & Overall \\
\hline $\begin{array}{l}\text { CIB-Coagulation Intervention Brigade (LFB } \\
\text { Biomedicaments) }\end{array}$ & 1.40 & 1.25 & 2.50 & 1.21 & 1.25 & 1.52 \\
\hline Leukemia Disease (Bedieman) & 1.50 & 1.50 & 1.33 & 1.71 & 1.13 & 1.43 \\
\hline $\begin{array}{l}\text { Recetas y consejos para combatir la anemia (App } \\
\text { Free Enjoy) }\end{array}$ & 1.60 & 1.50 & 1.50 & 1.43 & 1.00 & 1.41 \\
\hline Leukemia: Symptoms And Treatment (The Reyv) & 1.50 & 1.75 & 1.17 & 1.43 & 1.13 & 1.39 \\
\hline $\begin{array}{l}\text { Myeloma Cancer Guide (Everyone Learning } \\
\text { Apps) }\end{array}$ & 1.20 & 1.75 & 1.50 & 1.50 & 1.00 & 1.39 \\
\hline Leukemia Disease Treatment (Woochi Developer) & 1.40 & 1.50 & 1.17 & 1.43 & 1.25 & 1.35 \\
\hline Blood Cancer (Digital Planete Space) & 1.40 & 1.71 & 1.17 & 1.29 & 1.00 & 1.31 \\
\hline How To Cure Leukemia (Apps How To Apps) & 1.10 & 1.75 & 1.17 & 1.14 & 1.00 & 1.23 \\
\hline Blood Cancer tips (Free Apps For Everyone) & 1.00 & 1.63 & 1.17 & 1.14 & 1.00 & 1.19 \\
\hline Blood-Count Reader free (Yurii Shevchenko) & 1.80 & 1.13 & 1.00 & 1.00 & 1.00 & 1.19 \\
\hline
\end{tabular}

Comparison by app distribution platform (Apple App Store and Android Play Store) revealed a mean MARS score of 3.85 (SD $0.35)$ for apps developed for $\mathrm{iOS}(\mathrm{n}=34)$ and 2.67 (SD 0.30) for apps developed for Android ( $\mathrm{n}=62)$, resulting in a statistically significant difference $(P<.001)$. Apps whose development had been supported by a health organization obtained better scores (mean 3.75, SD 0.29; $\mathrm{n}=23$ ) than those that had not (mean 2.78, SD $0.31 ; \mathrm{n}=65 ; P<.001)$. Finally, another statistically significant difference $(P=.049)$ was found when the overall MARS scores were analyzed considering whether the apps were free (mean 2.97, SD 0.30; $\mathrm{n}=82$ ) or required payment (mean 3.92, SD 0.29; $\mathrm{n}=6 ; P=.049)$. The comparison by different characteristics is shown in Table 6.

The mean $\kappa$ coefficient score for the five MARS domains was 0.78 . $\kappa$ values between 0.61 and 0.81 indicate that interrater agreement between the 2 reviewers was substantial. The only item with a score less than 0.61 was ease of use (Table 7).

Table 6. Results of the Mobile App Rating Scale evaluation: comparison by different characteristics.

\begin{tabular}{|c|c|c|c|c|c|c|c|c|c|}
\hline \multirow[t]{2}{*}{ Category } & \multicolumn{3}{|c|}{ Operating system } & \multicolumn{3}{|l|}{ Developer } & \multicolumn{3}{|l|}{ Cost } \\
\hline & $\begin{array}{l}\text { Android } \\
(\mathrm{n}=62), \\
\text { score }\end{array}$ & $\begin{array}{l}\text { iOS } \\
(n=34), \\
\text { score }\end{array}$ & $P$ value & $\begin{array}{l}\text { No health organiza- } \\
\text { tion }(n=65) \text {, score }\end{array}$ & $\begin{array}{l}\text { Health organiza- } \\
\text { tion }(n=23) \text {, score }\end{array}$ & $P$ value & $\begin{array}{l}\text { Free } \\
(\mathrm{n}=82), \\
\text { score }\end{array}$ & $\begin{array}{l}\text { Payment } \\
(n=6), \\
\text { score }\end{array}$ & $P$ value \\
\hline Engagement & 2.59 & 4.16 & $<.001$ & 2.76 & 3.88 & $<.001$ & 2.98 & 4.12 & .04 \\
\hline Functionality & 3.09 & 4.01 & $<.001$ & 3.23 & 4.02 & .002 & 3.38 & 4.29 & .04 \\
\hline Aesthetics & 2.66 & 3.91 & $<.001$ & 2.82 & 3.90 & .002 & 3.04 & 3.89 & .10 \\
\hline Information & 2.64 & 3.52 & $<.001$ & 2.70 & 3.67 & $<.001$ & 2.90 & 3.70 & .08 \\
\hline Subjective quality & 2.26 & 3.34 & $<.001$ & 2.37 & 3.29 & .002 & 2.54 & 3.58 & .052 \\
\hline Overall & 2.67 & 3.85 & $<.001$ & 2.78 & 3.75 & $<.001$ & 2.97 & 3.92 & .049 \\
\hline
\end{tabular}


Table 7. Kappa score and interrater reliability for the Mobile App Rating Scale domains.

\begin{tabular}{|c|c|c|}
\hline Domain & Weighted Cohen $\kappa$ & Agreement, $\%$ \\
\hline Engagement & 0.82 & 93.1 \\
\hline Entertainment & 0.63 & 86.7 \\
\hline Interest & 0.72 & 90.4 \\
\hline Customization & 0.90 & 95.2 \\
\hline Interactivity & 0.84 & 92.6 \\
\hline Target group & 0.73 & 90.4 \\
\hline Functionality & 0.69 & 90.6 \\
\hline Performance & 0.67 & 88.5 \\
\hline Ease of use & 0.54 & 87.1 \\
\hline Navigation & 0.64 & 87.9 \\
\hline Gestural design & 0.71 & 90.3 \\
\hline Aesthetics & 0.80 & 93.1 \\
\hline Layout & 0.76 & 91.7 \\
\hline Graphics & 0.76 & 90.7 \\
\hline Visual appeal & 0.78 & 92.4 \\
\hline Information & 0.80 & 93.5 \\
\hline Accuracy of the app in the description (Apple App Store and Android Play Store) & 0.77 & 93.1 \\
\hline Goals & 0.78 & 92.8 \\
\hline Quality of information & 0.73 & 91.0 \\
\hline Quantity of information & 0.67 & 88.6 \\
\hline Visual information & 0.63 & 86.4 \\
\hline Evidence base & 0.91 & 96.3 \\
\hline Credibility & 0.84 & 94.3 \\
\hline Subjective quality & 0.80 & 92.8 \\
\hline Would you recommend this app to people who might benefit from it? & 0.78 & 91.5 \\
\hline Would you pay for this app? & 0.86 & 94.2 \\
\hline How many times do you think you would use this app in the next 12 months if it was relevant to you? & 0.77 & 90.3 \\
\hline What is your overall star rating of the app? & 0.79 & 92.2 \\
\hline
\end{tabular}

\section{Discussion}

\section{Principal Findings}

This is the first study to provide a systematic search and ranking of apps for patients diagnosed with hematological conditions available in the Apple App Store and Android Play Store, using the MARS as a standardized methodology for the classification, assessment, and validation of these apps.

We found that there were more apps available in the Android Play Store than in the Apple App Store, as mentioned in other studies [8,11,24], which can imply that uploading an app into the Android Play Store is an easier process. We observed that almost half of the apps $(43 / 88,49 \%)$ had been updated in the last year, as previously reported [25]. Considering hematology as a medical field that is constantly growing in complexity and extending its therapeutic arsenal, this low rate of app content actualization is insufficient [26].
Of 88 apps, only 23 (26\%) were designed with the participation of some kind of health organization. The absence of health care professionals in the development of health apps continues to be raised time and time again. Amor-García et al [11] observed that only $15.2 \%$ of apps for patients with genitourinary cancer involved health professionals in their design process. When reviewing apps for medication management, Tabi et al [27] observed a similar result (14.6\%). It would seem crucial that health care professionals be involved in the creation of medical apps; however, this scarcely happens. Moreover, the fact that most health-related apps are free favors accessibility [27].

Our results expose the high prevalence of informative apps $(60 / 88,68 \%)$, as reported by other authors $[6,11]$. The majority of these apps provide generic data about one or several pathologies, including symptoms, diagnostics, and treatment, focusing solely on education. One-third of the total of informative apps is intended for patients with anemia, which highlights the interest in anemia self-management, as it is the 
most common blood disorder globally [18]. Preventive apps are less numerous $(23 / 88,26 \%)$, although their quality and performance are significantly higher. These apps focus on handling the pathology after diagnosis, allowing for treatment and laboratory values tracking and recording of symptoms and adverse events. We found these types of apps the most appropriate and useful for patients with hematological conditions because many blood conditions require chronic and complex pharmacologic treatment [28,29]. Only 5 diagnostic apps were evaluated. It is worth mentioning STB-Stop The Bleed, an app designed to help anyone learn how to safely and effectively deal with life-threatening bleeding, which has demonstrated the potential of mobile apps in emergency scenarios [30]. The other 4 diagnostic apps are screening tools based on hematological parameters, questionnaires, and gene traits. Its objectives are to predict blood groups or certain hereditary pathologies, such as hemophilia or thalassemia. The main limitation is again the lack of evidence-based content, which in this case could mislead patients into not seeking professional advice. The potential of apps to be implemented as remote diagnostic tools for hematological conditions is very high. This is the case of AnemoCheck Mobile, an app that estimates hemoglobin levels by analyzing the color of fingernail beds and detects anemia, serving as a completely noninvasive anemia screening tool [31].

The MARS has demonstrated its potential as a simple, reliable, and flexible health care app-quality rating scale [21]. It analyzes the quality of an app by evaluating 23 items, grouped into 5 domains, and rating on a 5-point scale. Our study showed a mean score of 3.03, considering a score of 3 as acceptable. This result is similar to the scores showed by other authors using the MARS to evaluate health apps for other conditions. The mean score found by Salazar et al [5] for apps designed for chronic pain management was 3.17, and Kwan et al [6] showed a mean score of 3.48 for apps targeted at patients with spondyloarthritis, out of 18 and 5 apps evaluated, respectively. Knitza et al [24] reviewed 28 rheumatology apps and obtained an overall MARS score of 3.85. The median overall MARS score of the analysis of 34 apps targeted toward supporting heart failure symptom monitoring was 3.4 [32]. In a larger sample study, Amor-García et al [11] evaluated 46 apps for patients with genitourinary cancers and found a mean score of 2.98. It is worth noting that our study encompasses a higher number of apps evaluated than any of the studies cited. Thus, the overall quality of health apps in digital platforms is moderate, and there remains considerable scope for improvement. Of the 88 apps, 41 (47\%) hematological apps obtained a score of at least 3 points, meaning that more than half of the apps for hematological conditions do not meet acceptable criteria for quality and content. Moreover, of the 88 apps, only $28(32 \%)$ exceeded 4 points in the overall score.

MARS ratings ranged from 1.19 (Blood Count Reader) to 4.86 (Multiple Myeloma Manager), indicating the highly inconsistent quality of apps. The apps with the highest scored were Multiple Myeloma Manager, Hodgkin Lymphoma Manager, Focus On Lymphoma, ALL Manager, and CLL Manager. All of them were exclusive to the Apple App Store, except Focus On Lymphoma, which was available in both platforms. These apps showed high scores in the engagement and functionality domains. The main characteristic that defines these top-rated apps was the active patient participation, offering wide treatment and symptom monitoring options, reminders, and schedules edition. The five apps with the highest score had a plain preventive purpose, whereas informative apps scored lower on the MARS despite being more frequent.

The comparison by operating system showed a statistically significant difference favoring iOS apps over Android apps in all 5 MARS domains, a tendency that has been observed in a similar evaluation about genitourinary apps [11]. The reason could be that the Apple App Store has stricter standards to include apps.

Although we observed that only $26 \%$ (23/88) of the apps involved the participation of health professionals in their design, their quality was significantly higher. The lack of health professional involvement is a constant that has already been highlighted by several authors, expressing their concern about app content and compromising patient safety [33-35]. However, 4 of the best apps (Multiple Myeloma Manager, Hodgkin Lymphoma Manager, ALL Manager, and CLL Manager) were developed by @Point of Care, a platform consisting of nonmedical stakeholders and dedicated to creating medical apps for patients and clinicians. @ Point of Care has designed apps focused on diverse pathologies, some of them obtaining considerably high MARS scores in other studies similar to ours [11]. The analysis by cost revealed another statistically significant difference, positioning payment apps ahead of free apps in terms of quality, although the fact that only 6 hematological apps were not free and all of them were developed for iOS can destabilize the comparison.

Functionality was the domain that scored the highest on the MARS test, as described by other authors $[11,36]$. This implies that the apps are easy to navigate and efficient. Leaving subjective quality aside, engagement and information were the domains with the lowest MARS scores. Engagement reflects the capacity of the app to be personalized by the user. Patients usually search for a health app that allows for medication management, clinical and analytical parameter register, and symptom tracking [28,37]. Patients with hematological conditions would benefit significantly from this type of assistance, as several blood conditions demand constant patient monitoring and high adherence to treatment for a better health outcome [18,30]. My INR, INR Care, and Diario de INR are apps that allow anticoagulated patients to record and track their international normalized ratio readings and antivitamin $\mathrm{K}$ dosages. They could help improve adherence and avoid potential complications, such as the risk of bleeding or clots. HaemActive is a fitness app especially tailored to patients with hemophilia, who require special exercises that imply a minimal risk of bleeding. The app includes weekly training planning, explainer videos, and easy customization. In addition, patients expressed their interest in using health apps to communicate with their physicians [28,38]. Concerning the information domain, there is 1 specific item assessing the evidence base, which explores the extent to which the app has been scientifically tested. However, this item was excluded from all calculations, as no clinical studies to support the effectiveness and safety of any of the apps could be found. Thus, empirical studies should be conducted for apps to determine their clinical impact on 
outcomes for patients diagnosed with hematological conditions [13].

\section{Recommendations for Health App Development}

The number of health apps available and studies reviewing their quality is steadily growing, which will help health professionals to recommend apps to patients. This activity acquires even further relevance, considering the still little control from regulatory authorities over health app development. We have observed that the main issues that need to be addressed when designing health apps are as follows: no participation of health organizations in app development, questionable sources of information, and deficient interactivity and personalization options [35]. Production of medical apps from nonmedical stakeholders has benefits in terms of creativity in the design of apps. However, it must be combined with clinician assistance to boost the credibility of medical information with such apps. Concerning patients with hematological conditions, registering analytical information, treatment prescribed, and symptoms is highly recommendable for apps to help them in their care.

\section{Limitations}

First, only apps available in the Android Play Store and Apple App Store, with contents in English or Spanish and accessed from a Spanish IP address were included, assuming the possibility of having missed some other apps dedicated to hematological conditions. Another limitation could be that app quality was assessed using the MARS, which is limited by the subjectivity of the evaluators. Nevertheless, this issue is partially addressed by the high interrater reliability of the data analyzed by the 2 independent researchers. We believe that this evaluation should allow health care professionals and patients to identify which apps meet minimum standards of quality and safety in their content.

\section{Conclusions}

We provide the first systematic review of apps related to hematological conditions, identifying 88 apps and rating them using the MARS. The study shows great heterogeneity among their quality. Many of these apps emerge as tools for consulting information, being the most frequent functionality, although not the highest rated. A very small number of them offer a comprehensive self-management approach incorporating evidence-based strategies. Only 26\% (23/88) of the apps were developed with the assistance of health care professionals. The top 5 rated apps-Multiple Myeloma Manager, Hodgkin Lymphoma Manager, Focus On Lymphoma, ALL Manager, and CLL Manager - allowed for active patient participation and app personalization. Higher scores in quality were observed in iOS apps, apps developed by health organizations, and payment apps.

\section{Conflicts of Interest}

None declared.

\section{References}

1. World Health Organization. mHealth Economics 2017: current status and future trends in mobile health. Research2Guidance. URL: https://research2guidance.com/product/mhealth-economics-2017-current-status-and-future-trends-in-mobile-health/ [accessed 2021-05-05]

2. Number of mHealth apps available in the Google Play Store from 1st quarter 2015 to 1st quarter 2021. Statista. 2021. URL: https://www.statista.com/statistics/779919/health-apps-available-google-play-worldwide/ [accessed 2021-07-07]

3. Kebede MM, Pischke CR. Popular diabetes apps and the impact of diabetes app use on self-care behaviour: a survey among the digital community of persons with diabetes on social media. Front Endocrinol (Lausanne) 2019;10:135 [FREE Full text] [doi: 10.3389/fendo.2019.00135] [Medline: $\underline{\text { 30881349] }}$

4. Kalhori SR, Hemmat M, Noori T, Heydarian S, Katigari MR. Quality evaluation of English mobile applications for gestational diabetes: app review using mobile application rating scale (MARS). Curr Diabetes Rev 2021;7(2):161-168. [doi: 10.2174/1573399816666200703181438] [Medline: 32619173]

5. Salazar A, de Sola H, Failde I, Moral-Munoz JA. Measuring the quality of mobile apps for the management of pain: systematic search and evaluation using the mobile app rating scale. JMIR Mhealth Uhealth 2018 Oct 25;6(10):e10718 [FREE Full text] [doi: 10.2196/10718] [Medline: $\underline{\text { 30361196] }}$

6. Kwan YH, Ong WJ, Xiong M, Leung YY, Phang JK, Wang CT, et al. Evaluation of mobile apps targeted at patients with spondyloarthritis for disease monitoring: systematic app search. JMIR Mhealth Uhealth 2019 Oct 28;7(10):e14753 [FREE Full text] [doi: 10.2196/14753] [Medline: 31661080]

7. Terhorst Y, Rathner E, Baumeister H, Sander L. «Hilfe aus dem App-Store?»: Eine systematische Übersichtsarbeit und Evaluation von Apps zur Anwendung bei Depressionen. Verhaltenstherapie 2018 May 8;28(2):101-112. [doi: 10.1159/000481692]

8. Collado-Borrell R, Escudero-Vilaplana V, Villanueva-Bueno C, Herranz-Alonso A, Sanjurjo-Saez M. Features and functionalities of smartphone apps related to COVID-19: systematic search in app stores and content analysis. J Med Internet Res 2020 Aug 25;22(8):e20334 [FREE Full text] [doi: 10.2196/20334] [Medline: 32614777]

9. Singh HJ, Couch D, Yap K. Mobile health apps that help with COVID-19 management: scoping review. JMIR Nurs 2020;3(1):e20596 [FREE Full text] [doi: 10.2196/20596] [Medline: 32897271]

10. Salehinejad S, Kalhori SR, Gohari SH, Bahaadinbeigy K, Fatehi F. A review and content analysis of national apps for COVID-19 management using Mobile Application Rating Scale (MARS). Inform Health Soc Care 2021 Mar 02;46(1):42-55. [doi: 10.1080/17538157.2020.1837838] [Medline: 33164594] 
11. Amor-García MA, Collado-Borrell R, Escudero-Vilaplana V, Melgarejo-Ortuño A, Herranz-Alonso A, Arija A, et al. Assessing apps for patients with genitourinary tumors using the mobile application rating scale (MARS): systematic search in app stores and content analysis. JMIR Mhealth Uhealth $2020 \mathrm{Jul}$ 23;8(7):e17609 [FREE Full text] [doi: 10.2196/17609] [Medline: 32706737]

12. Ali EE, Chan SS, Leow JL, Chew L, Yap KY. User acceptance of an app-based adherence intervention: perspectives from patients taking oral anticancer medications. J Oncol Pharm Pract 2019 Mar;25(2):390-397 [FREE Full text] [doi: 10.1177/1078155218778106] [Medline: 29792123]

13. Jongerius C, Russo S, Mazzocco K, Pravettoni G. Research-tested mobile apps for breast cancer care: systematic review. JMIR Mhealth Uhealth 2019 Feb 11;7(2):e10930 [FREE Full text] [doi: 10.2196/10930] [Medline: $\underline{30741644]}$

14. Burns R, Leal J, Sullivan R, Luengo-Fernandez R. Economic burden of malignant blood disorders across Europe: a population-based cost analysis. Lancet Haematol 2016 Aug;3(8):362-370. [doi: 10.1016/S2352-3026(16)30062-X] [Medline: 27476787]

15. Boström EA, Lira-Junior R. Non-malignant blood disorders and their impact on oral health: an overview. Curr Oral Health Rep 2019 Apr 24;6(2):161-168. [doi: 10.1007/s40496-019-0211-9]

16. Atrash HK, Parker CS. The public health response to blood disorders. Am J Prev Med 2010 Apr;38(4 Suppl):451-455. [doi: 10.1016/j.amepre.2010.01.006] [Medline: 20331942]

17. Boccalandro EA, Dallari G, Mannucci PM. Telemedicine and telerehabilitation: current and forthcoming applications in haemophilia. Blood Transfus 2019 Sep;17(5):385-390 [FREE Full text] [doi: 10.2450/2019.0218-18] [Medline: 30747703 ]

18. Mann D, Riddell L, Lim K, Byrne LK, Nowson C, Rigo M, et al. Mobile phone app aimed at improving iron intake and bioavailability in premenopausal women: a qualitative evaluation. JMIR Mhealth Uhealth 2015 Sep 28;3(3):e92 [FREE Full text] [doi: 10.2196/mhealth.4300] [Medline: 26416479]

19. Agarwal P, Gordon D, Griffith J, Kithulegoda N, Witteman HO, Sacha Bhatia R, et al. Assessing the quality of mobile applications in chronic disease management: a scoping review. NPJ Digit Med 2021 Mar 10;4(1):46 [REEE Full text] [doi: 10.1038/s41746-021-00410-x] [Medline: 33692488]

20. Terhorst Y, Philippi P, Sander LB, Schultchen D, Paganini S, Bardus M, et al. Validation of the Mobile Application Rating Scale (MARS). PLoS One 2020;15(11):e0241480 [FREE Full text] [doi: 10.1371/journal.pone.0241480] [Medline: 33137123]

21. Stoyanov SR, Hides L, Kavanagh DJ, Zelenko O, Tjondronegoro D, Mani M. Mobile app rating scale: a new tool for assessing the quality of health mobile apps. JMIR Mhealth Uhealth 2015;3(1):e27 [FREE Full text] [doi: 10.2196/mhealth.3422] [Medline: 25760773]

22. Moher D, Shamseer L, Clarke M, Ghersi D, Liberati A, Petticrew M, et al. Preferred reporting items for systematic review and meta-analysis protocols (PRISMA-P) 2015 statement. Syst Rev 2015 Jan;4:1 [FREE Full text] [doi: 10.1186/2046-4053-4-1] [Medline: 25554246]

23. McHugh ML. Interrater reliability: the kappa statistic. Biochem Med (Zagreb) 2012;22(3):276-282 [FREE Full text] [Medline: 23092060]

24. Knitza J, Tascilar K, Messner E, Meyer M, Vossen D, Pulla A, et al. German mobile apps in rheumatology: review and analysis using the mobile application rating scale (MARS). JMIR Mhealth Uhealth 2019 Aug 05;7(8):e14991 [FREE Full text] [doi: 10.2196/14991] [Medline: 31381501]

25. Collado-Borrell R, Escudero-Vilaplana V, Ribed-Sánchez A, Ibáñez-García S, Herranz-Alonso A, Sanjurjo-Sáez M. Smartphone applications for cancer patients; what we know about them? Farm Hosp 2016 Jan 01;40(1):25-35 [FREE Full text] [doi: 10.7399/fh.2016.40.1.8993] [Medline: 26882831]

26. Finnes HD. 2019-2020 Drug updates in hematologic malignancies. J Adv Pract Oncol 2021 Apr;12(3):279-283 [FREE Full text] [doi: 10.6004/jadpro.2021.12.3.12] [Medline: 34084575]

27. Tabi K, Randhawa AS, Choi F, Mithani Z, Albers F, Schnieder M, et al. Mobile apps for medication management: review and analysis. JMIR Mhealth Uhealth 2019 Sep 11;7(9):e13608 [FREE Full text] [doi: 10.2196/13608] [Medline: 31512580 ]

28. Collado-Borrell R, Escudero-Vilaplana V, Calles A, Garcia-Martin E, Marzal-Alfaro B, Gonzalez-Haba E, et al. Oncology patient interest in the use of new technologies to manage their disease: cross-sectional survey. J Med Internet Res 2018 Oct 23;20(10):e11006 [FREE Full text] [doi: 10.2196/11006] [Medline: 30355554]

29. Shah N, Jonassaint J, De CL. Patients welcome the sickle cell disease mobile application to record symptoms via technology (SMART). Hemoglobin 2014;38(2):99-103. [doi: 10.3109/03630269.2014.880716] [Medline: 24512633]

30. Roncero AP, Marques G, Sainz-De-Abajo B, Martín-Rodríguez F, Vegas CD, Garcia-Zapirain B, et al. Mobile health apps for medical emergencies: systematic review. JMIR Mhealth Uhealth 2020 Dec 11;8(12):e18513 [FREE Full text] [doi: 10.2196/18513] [Medline: 33306037]

31. Mannino RG, Myers DR, Tyburski EA, Caruso C, Boudreaux J, Leong T, et al. Smartphone app for non-invasive detection of anemia using only patient-sourced photos. Nat Commun 2018 Dec 04;9(1):4924 [FREE Full text] [doi: 10.1038/s41467-018-07262-2] [Medline: 30514831]

32. Masterson CR, Maurer MS, Reading M, Hiraldo G, Hickey KT, Iribarren S. Review and analysis of existing mobile phone apps to support heart failure symptom monitoring and self-care management using the mobile application rating scale (MARS). JMIR Mhealth Uhealth 2016 Jun 14;4(2):e74. [doi: 10.2196/mhealth.5882] [Medline: 27302310] 
33. Tomlinson M, Rotheram-Borus MJ, Swartz L, Tsai AC. Scaling up mHealth: where is the evidence? PLoS Med 2013;10(2):e1001382 [FREE Full text] [doi: 10.1371/journal.pmed.1001382] [Medline: 23424286]

34. Biviji R, Vest JR, Dixon BE, Cullen T, Harle CA. Factors related to user ratings and user downloads of mobile apps for maternal and infant health: cross-sectional study. JMIR Mhealth Uhealth 2020 Jan 24;8(1):e15663 [FREE Full text] [doi: 10.2196/15663] [Medline: 32012107$]$

35. Morillo-Verdugo R, Margusino-Framiñán L, Monte-Boquet E, Morell-Baladrón A, Barreda-Hernández D, Rey-Piñeiro $\mathrm{XM}$, et al. Spanish society of hospital pharmacy position statement on telepharmacy: recommendations for its implementation and development. Farm Hosp 2020 Jul 01;44(4):174-181 [FREE Full text] [doi: 10.7399/fh.11515] [Medline: $\underline{32646349]}$

36. Jupp JC, Sultani H, Cooper CA, Peterson KA, Truong TH. Evaluation of mobile phone applications to support medication adherence and symptom management in oncology patients. Pediatr Blood Cancer 2018 Nov;65(11):e27278. [doi: 10.1002/pbc.27278] [Medline: 29943893]

37. Martínez-Bautista MJ, Mangues-Bafalluy I, Cajaraville-Ordoñana G, Carreras-Soler MJ, Clopés-Estela A, Moreno-Martínez E. Survey of oncohematological pharmaceutical care situation in Spain. Farm Hosp 2019 Nov 01;43(6):194-201 [FREE Full text] [doi: 10.7399/fh.11239] [Medline: $\underline{\text { 31705643] }}$

38. Collado-Borrell R, Escudero-Vilaplana V, Ribed A, Gonzalez-Anleo C, Martin-Conde M, Romero-Jimenez R, et al. Effect of a mobile app for the pharmacotherapeutic follow-up of patients with cancer on their health outcomes: quasi-experimental study. JMIR Mhealth Uhealth 2020 Oct 16;8(10):e20480 [FREE Full text] [doi: 10.2196/20480] [Medline: $\underline{33064100]}$

\author{
Abbreviations \\ MARS: Mobile App Rating Scale \\ PRISMA-P: Preferred Reporting Items for Systematic Review and Meta-Analysis Protocols
}

\author{
Edited by L Buis; submitted 03.09.21; peer-reviewed by R Romero-Jimenez, B Chaudhry; comments to author 18.10.21; revised \\ version received 28.10.21; accepted 10.12.21; published 16.02.22 \\ Please cite as: \\ Narrillos-Moraza Á, Gómez-Martínez-Sagrera P, Amor-García MÁ, Escudero-Vilaplana V, Collado-Borrell R, Villanueva-Bueno C, \\ Gómez-Centurión I, Herranz-Alonso A, Sanjurjo-Sáez M \\ Mobile Apps for Hematological Conditions: Review and Content Analysis Using the Mobile App Rating Scale \\ JMIR Mhealth Uhealth 2022;10(2):e32826 \\ URL: https://mhealth.jmir.org/2022/2/e32826 \\ doi: $10.2196 / 32826$ \\ PMID:
}

(CÁlvaro Narrillos-Moraza, Patricia Gómez-Martínez-Sagrera, Miguel Ángel Amor-García, Vicente Escudero-Vilaplana, Roberto Collado-Borrell, Cristina Villanueva-Bueno, Ignacio Gómez-Centurión, Ana Herranz-Alonso, María Sanjurjo-Sáez. Originally published in JMIR mHealth and uHealth (https://mhealth.jmir.org), 16.02.2022. This is an open-access article distributed under the terms of the Creative Commons Attribution License (https://creativecommons.org/licenses/by/4.0/), which permits unrestricted use, distribution, and reproduction in any medium, provided the original work, first published in JMIR mHealth and uHealth, is properly cited. The complete bibliographic information, a link to the original publication on https://mhealth.jmir.org/, as well as this copyright and license information must be included. 